\title{
Ligand Exchange and Impurity Doping in 2D CdSe Nanoplatelet Thin Films and Their Applications
}

\author{
Woo Seok Lee, Yoon-Gu Kang, Manoj Sharma, Yong Min Lee, Sanghyun Jeon, \\ Ashma Sharma, Hilmi Volkan Demir,* Myung Joon Han,* Weon-Kyu Koh,* \\ and Soong Ju Oh*
}

The effects of halide-ligand exchange and $\mathrm{Cu}$ and $\mathrm{Ag}$ doping are studied on structural, optical, and electrical properties of four monolayer CdSe nanoplatelet (NPL) and NPL thin films. Combinational study shows that $\mathrm{NH}_{4} \mathrm{Cl}$-treatment on CdSe NPL and NPL thin films show tetragonal lattice distortion of NPL, side-to-side attachment between NPLs, bathochromic shift in absorption spectra, and complete quenching of band-edge and dopantinduced emissions. First-principle calculations reveal that $\mathrm{Cl}$ creates states below valence band maximum while $\mathrm{Ag}$ and $\mathrm{Cu}$ dopants create acceptor-like states, explaining the change of their optical property. Field-effect transistors are fabricated to investigate the effect of doping and reduced interplatelet distance on electrical properties of CdSe NPL thin films, demonstrating $\mathrm{Cu}$ and $\mathrm{Ag}$ dopants mitigate $\mathrm{n}$-type character of CdSe NPL thin films. Temperature-dependent electrical characterization is conducted to further understand charge transport behavior depending on the existence of dopants. This work provides scientific information on the influence of surface chemistry and impurity doping on quantum confined semiconductors and new directions for the design of high-performance nanomaterial-based electronic and optoelectronic devices.

\section{Introduction}

2D colloidal nanoplatelets (NPLs) have been attracting huge attention for electronic and optoelectronic applications because of solution processability and precisely controllable electronic and optical properties depending on their size, composition and surface chemistry. ${ }^{[1-5]}$ In particular, their highly anisotropic thin structure offers exceptional excitonic features such as narrow absorption and emission line widths because of strong $1 \mathrm{D}$ quantum confinement and reduced dielectric constant. ${ }^{[6-9]}$ Furthermore, exciton transition dipole in NPLs is fully oriented within the plane, enabling the improvement of external efficiency of NPL based optoelectronic devices by optimizing their assembly. ${ }^{[10]}$ There have been many efforts to atomically adjust thickness of NPLs and control the orientation and assembly of NPLs to fully exploit their exceptional excitonic features. ${ }^{[10-13]}$
W. S. Lee, S. Jeon, S. J. Oh

Department of Materials Science and Engineering

Korea University

Seoul 02841, Republic of Korea

E-mail: sjoh1982@korea.ac.kr

W. S. Lee

Department of Materials Science and Engineering

Massachusetts Institute of Technology

Cambridge, MA 02139, USA

Y.-G. Kang, M. J. Han

Department of Physics

Korea Advanced Institute of Science and Technology

Daejeon 34141, Republic of Korea

E-mail:mj.han@kaist.ac.kr

M. Sharma, A. Sharma, H. V. Demir, W.-K. Koh

LUMINOUS! Centre of Excellence for Semiconductor

Lighting and Displays

School of Electrical and Electronic Engineering

Nanyang Technological University

50 Nanyang Avenue, Singapore 639798, Singapore

E-mail:hvdemir@ntu.edu.sg; revolu77@snu.ac.kr
M. Sharma

ARC Centre of Excellence in Exciton Science

Department of Materials Science and Engineering

Monash University

Clayton Campus, Melbourne, Victoria 3800, Australia

Y. M. Lee

Department of Semiconductor Systems Engineering

Korea University

Seoul 02841, Republic of Korea

H. V. Demir

School of Physical and Mathematical Sciences

Nanyang Technological University

Singapore 639798, Singapore

H. V. Demir

Department of Electrical and Electronics Engineering

and Department of Physics

UNAM-Institute of Materials Science and Nanotechnology

Bilkent University

Bilkent, Ankara 06800, Turkey

The ORCID identification number(s) for the author(s) of this article can be found under https://doi.org/10.1002/aelm.202100739.

DOI: 10.1002/aelm.202100739 
While the excitonic characteristics of such NPLs made of CdSe have been widely studied, their charge carrier transport has been rarely investigated despite the importance of its understanding for the realization of high-performance electronic and optoelectronic devices. ${ }^{[14-17]}$ This is because as-synthesized NPLs are capped with long carbonic ligands, which leads to long interplatelet distance and prohibit efficient charge transport between NPLs. ${ }^{[1]}$ Surface ligand modification can be a simple and effective way to decrease interplatelet distance and to improve charge transport efficiency as frequently demonstrated in $0 \mathrm{D}$ or $1 \mathrm{D}$ nanocrystals. ${ }^{[18-22]}$ However, previous studies have only focused on structural and optical properties of 2D CdSe NPLs after ligand exchange. ${ }^{[23-29]}$

Impurity doping of semiconductor nanomaterials is scientifically important and technologically essential because impurity atoms can control electrical properties by providing extra electrons or holes as well as tune the colors of luminescence by forming mid-gap states that give rise to dopant-induced emission. ${ }^{[30-33]}$ For electrical doping, Luo et al. synthesized Ga-doped quantum dots and demonstrated enhanced conductivity. ${ }^{[34]}$ Lee et al. doped CdSe quantum dots with In by replacing original ligands with In-halide-complex ligands, increasing carrier concentration and mobility. ${ }^{[35]}$ In the case of optical doping, $\mathrm{Cu}$ and Ag have been known to form in-gap states in CdSe, which enables dopant-related broad emission. ${ }^{[36-38]}$ However, most of previous reports have only focused on the effects of $\mathrm{Cu}$ and $\mathrm{Ag}$ dopants on optical properties of CdSe nanomaterials and little has been reported about electrical properties and charge transport of $\mathrm{Cu}$ - or Ag-doped CdSe nanomaterials, especially in 2D NPL systems. ${ }^{[39,40]}$ Indeed, a systematic study for a comprehensive understanding of the influences of both ligand exchange and impurity doping on structural, optical and electrical properties is needed to make high-performance 2D NPL based electronic and optoelectronic devices.
In this work, we systematically studied the influences of ligand exchange and impurity doping on structural and electronic properties of four monolayer CdSe NPLs and their thin films using both experimental and theoretical methods. Pristine, Cu-doped and Ag-doped four monolayer CdSe NPLs were synthesized and halide-ligand exchange was conducted to adjust surface chemistry and interplatelet distance of NPLs. Side-to-side attachment between NPLs, tetragonal lattice distortion, bathochromic shift in absorption spectra, and complete quenching of their band-edge and dopant-induced emissions were observed and systematically discussed by correlating with each other. First-principle calculations were also conducted to fully understand these observations and support the discussion. Analysis of electrical properties and charge transport behaviors of CdSe NPL thin films with field-effect transistors (FETs) geometry show that $\mathrm{Cu}$ and $\mathrm{Ag}$ dopants mitigate n-type character of CdSe NPLs. Temperature-dependent electrical characterization of these CdSe NPL FETs was conducted to further understand the origin of different mobility and charge transport behavior depending on the existence of dopants. This work provides scientifically critical and technologically useful information about the effect of surface chemistry and doping on various properties of nanomaterials, paving the way for the realization of low-cost, high-performance electronic and optoelectronic applications based on quantum-confined materials.

\section{Results and Discussion}

Four monolayer (ML) of pristine, Cu-doped and Ag-doped CdSe nanoplateletes (NPLs) with a thickness of $1.2 \mathrm{~nm}$ were synthesized to have lengths and widths of 35.0 by $8.0 \mathrm{~nm}, 50.8$ by $8.0 \mathrm{~nm}$, and 36.0 by $7.0 \mathrm{~nm}$, respectively (Figure 1a). ${ }^{[41,42]}$ It was reported that $\mathrm{OH}$-ions or water content during synthesis
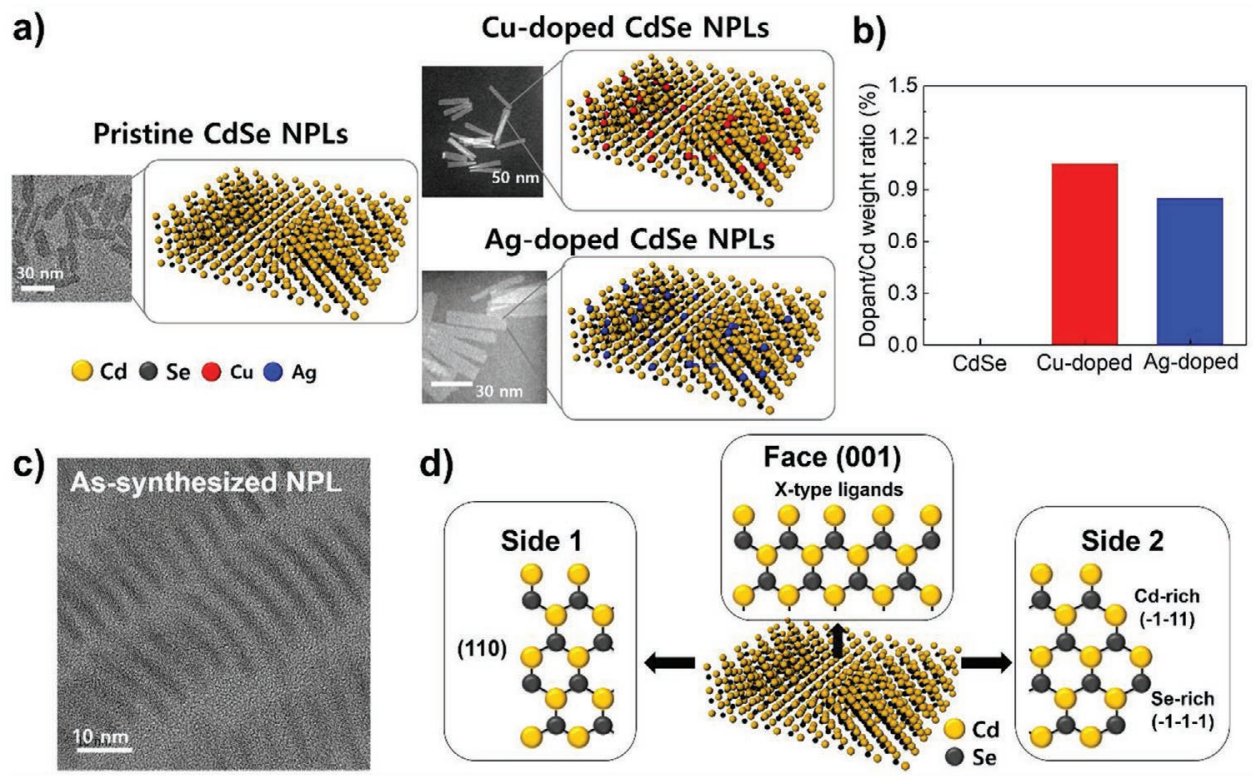

Figure 1. a) Schematics and TEM images of as-synthesized pristine, Cu-doped and Ag-doped CdSe NPLs with face-up configurations. b) Dopant/Cd weight ratio of pristine, Cu-doped and Ag-doped NPLs measured using ICP-MS. c) TEM images of as-synthesized pristine NPLs in side-up assembly with face-to-face stacking. d) tomic configurations of surface planes of as-synthesized CdSe NPL. 
a)

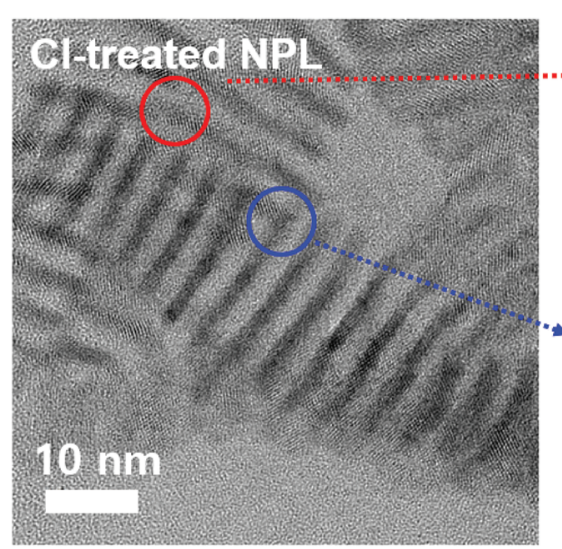

c)

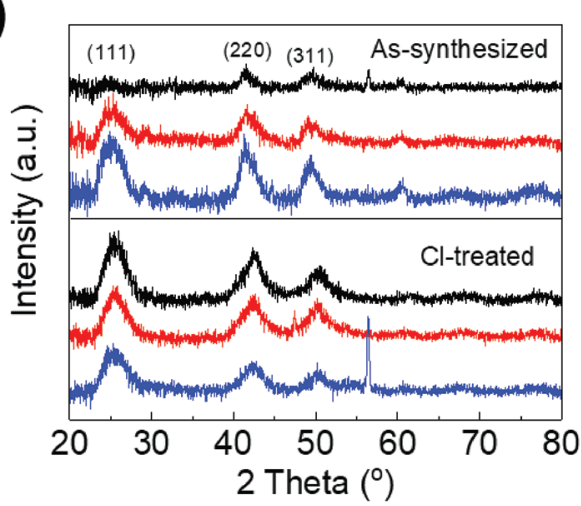

e)

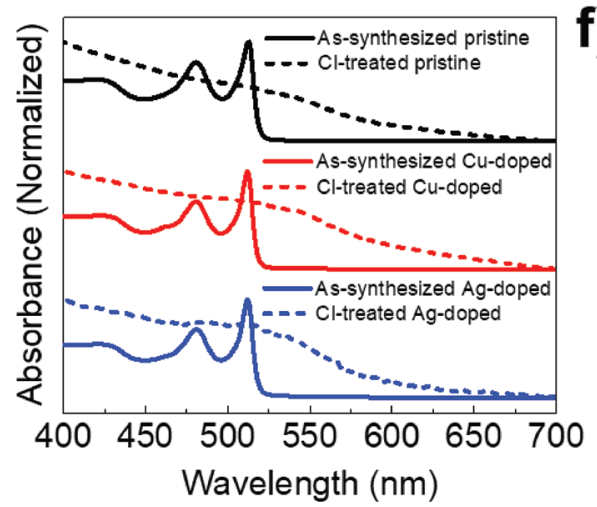

b)

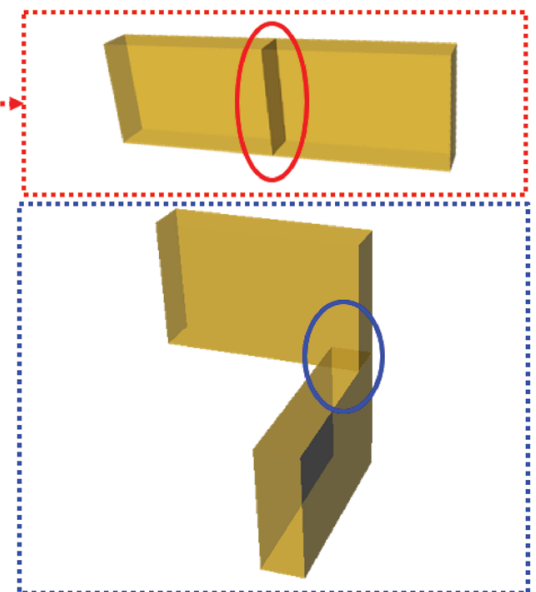

d)

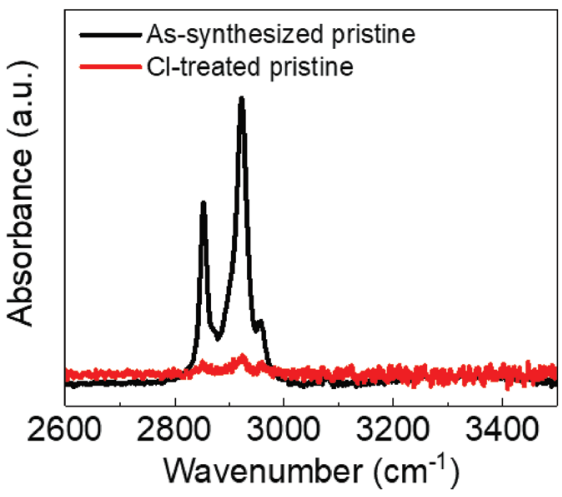

f)

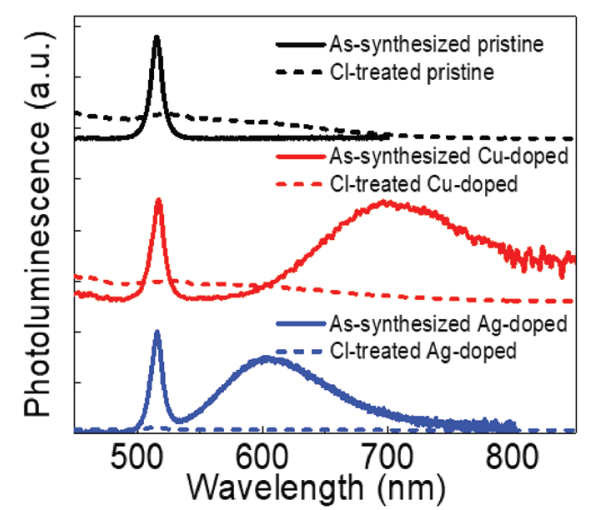

Figure 2. a) A TEM image of pristine CdSe NPLs and b) schematics of side-to-side attachment between NPLs after $\mathrm{NH}_{4} \mathrm{Cl}$-ligand exchange. c) $\mathrm{XRD}^{-}$ patterns of (top) as-synthesized and (bottom) $\mathrm{NH}_{4} \mathrm{Cl}$-treated (black) pristine, (red) $\mathrm{Cu}$ - doped, and (blue) Ag-doped CdSe $\mathrm{NPLs}$. Peaks at around $47.4^{\circ}$ and $56.4^{\circ}$ are originated by Si substrate. d) FTIR spectra of pristine CdSe NPLs (black) before and (red) after NH ${ }_{4} \mathrm{Cl}-$ ligand exchange. e) UV-vis absorption spectra of (black) pristine, (red) $\mathrm{Cu}$ - doped, and (blue) Ag-doped CdSe NPLs (solid line) before and (dotted line) after $\mathrm{NH}_{4} \mathrm{Cl}$-ligand exchange. f) $\mathrm{PL}$ spectra of (black) pristine, (red) Cu-doped, and (blue) Ag-doped CdSe NPLs (solid line) before and (dotted line) after $\mathrm{NH}_{4} \mathrm{Cl}$-ligand exchange.

could affect the aspect ratio of CdSe NPLs. ${ }^{[43]}$ However, the same amount of cadmium actate dihydrate was used in all synthesis of undoped and doped NPL, implying that the addition of dopant ions slightly changes the aspect ratio of NPLs as demonstrated in our previous reports. ${ }^{[41,44]}$ Inductively coupled plasma-mass spectrometry (ICP-MS) was conducted to measure the doping concentration of $\mathrm{Cu}$ - and Ag-doped CdSe NPLs, showing 1.05 weight $\%(\mathrm{Cu} / \mathrm{Cd})$ and 0.85 weight \% $(\mathrm{Ag} / \mathrm{Cd})$, respectively (Figure $1 \mathrm{~b}$ ). The NPL solutions were spin-coated onto pre-cleaned and (3-Mercaptopropyl)trimethoxysilane (MPTS)-treated substrates to form NPL thin films. ${ }^{[45]}$ The assynthesized NPLs were observed to preferentially form side-up assembly with face-to-face stacking in transmission electron microscopy (TEM) analysis (Figure 1c; Figure S1a,b, Supporting Information). It is well known that the top and bottom plane of as-synthesized zinc blende CdSe NPLs are Cd-terminated (001) plane and passivated by long organic ligands with X-type ligation (Figure 1d). In contrast, it is generally hard to distinguish 
whether the side plane is (100), (110), the combination of (-111) and (-1-1-1), or atomically rough because the side plane is composed of only a few atoms. ${ }^{[46-48]}$ However, TEM analysis shows that side-to-side distance between NPLs is over $2 \mathrm{~nm}$, implying that the side plane of NPL might be partially passivated by oleate ligands (Figure 1c; Figure S1a,b, Supporting Information). These long original ligands must be removed or replaced with short ligands to improve charge transport efficiency between NPLs for a charge transport study and electronic device applications. To achieve this, as-synthesized NPL thin films were immersed into $1 \times 10^{-3} \mathrm{M}$ solution of $\mathrm{NH}_{4} \mathrm{Cl}$ in acetone to replace long original ligands with short inorganic ligands of $\mathrm{Cl}^{-}$.

The structural, chemical, and optical properties of pristine, Cu-doped, and Ag-doped CdSe NPLs before and after ligand exchange with $\mathrm{NH}_{4} \mathrm{Cl}$ are shown in Figure 2. The morphological and structural change of NPLs after $\mathrm{NH}_{4} \mathrm{Cl}$-treatment were investigated using TEM (Figure 2a; Figure S1c,d, Supporting Information). After $\mathrm{NH}_{4} \mathrm{Cl}$-treatment, the structure and morphology of CdSe NPLs almost stayed similar to the original state. One interesting observation is that only side-to-side interplatelet distance between NPLs was significantly decreased and neighboring NPLs were even touched through sideto-side attachment, while face-to-face distance remained similar after the $\mathrm{NH}_{4} \mathrm{Cl}$-treatment (Figure 2a,b; and Figure S1c,d, Supporting Information). X-ray diffraction (XRD) analysis was conducted on NPL thin films to further investigate the structural property of CdSe NPL thin films before and after $\mathrm{NH}_{4} \mathrm{Cl}$-treatment (Figure 2c). As-synthesized CdSe NPL thin films showed characteristic XRD patterns of zinc blende crystal structure. It should be noted that as-synthesized zinc blende CdSe NPL has different in-plane and out-of-plane lattice parameters because of steric repulsion and Van der Waals attraction of ligand hydrocarbon tails, different ligand packing density and ligand head group induced surface reconstruction. ${ }^{[23,49,50]}$ For example, bimodal nature of the (220) reflection can be split into sharp and broad peaks, which correspond to interplanar distances in in-plane and out-of-plane directions, respectively (Figure S2a, Supporting Information). Positions of sharp and broad peaks were changed after $\mathrm{NH}_{4} \mathrm{Cl}$ treatment, indicating tetragonal lattice distortion (Figure S2b,c, Supporting Information).

Fourier-transform infrared spectroscopy (FTIR) and scanning electron microscopy with energy-dispersive X-ray measurement (SEM-EDS) were undertaken to investigate the surface chemistry of CdSe NPLs before and after $\mathrm{NH}_{4} \mathrm{Cl}$-treatment. Absorption peaks at around $2900 \mathrm{~cm}^{-1}$ corresponding to C-H stretch of original ligands were observed for as-synthesized NPL thin films (Figure 2d). These peaks were significantly decreased but still slightly remained after $\mathrm{NH}_{4} \mathrm{Cl}$-treatment, indicating that over the $90 \%$ of the original surface ligands were replaced with $\mathrm{Cl}^{-}$ligands as confirmed by EDS measurement (Figure S3, Supporting Information). It has been reported that top and bottom (001) plane of as-synthesized zinc blende are the most passivated with carboxylic acid ligands and are stable compared to side planes regardless of whether they are (100), (111), or the combination of (-1-11) and (-1-1-1)..$^{[46-48]}$ Therefore, original ligands weakly bonded to side plane were readily detached or replaced with $\mathrm{Cl}^{-}$during ligand exchange process, leading to a dramatic decrease of side-to-side distance or sideto-side attachment and formation of NPL networks, whereas residual original ligands on face plane have face-to-face distance maintained. These decreased side-to-side distance or sideto-side attachment significantly improve the charge transport efficiency between NPLs as will be discussed below.

Ultraviolet-visible (UV-vis) spectra of pristine, Cu-doped, and Ag-doped CdSe NPL thin films before and after $\mathrm{NH}_{4} \mathrm{Cl}$ treatment are shown in Figure 2e. As-synthesized pristine, Cu-doped, and Ag-doped CdSe NPL thin films exhibit an absorption peak at $513 \mathrm{~nm}$, corresponding to the heavy hole

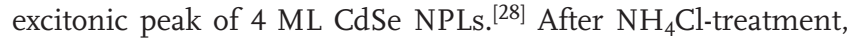
bathochromic shift and broadened absorption peaks were observed for all series of CdSe NPL thin films. Photoluminescence (PL) emission spectra are analyzed in Figure 2f. A narrow PL emission centered at $515 \mathrm{~nm}$ corresponding to band edge emission was observed for all series of as-synthesized CdSe NPL thin films. Broad emission centered at $653 \mathrm{~nm}$ and $632 \mathrm{~nm}$ was also observed for as-synthesized $\mathrm{Cu}$-doped and $\mathrm{Ag}$ doped CdSe PL thin films, respectively, which is corresponding to dopant-induced emission. ${ }^{[41,42]} \mathrm{After} \mathrm{NH}_{4} \mathrm{Cl}$-treatement, both band-edge emission and dopant-induced emission were significantly quenched.

The absorption peak shift and broadening could be attributed to two following reasons: 1 ) increased electronic coupling between NPLs and/or sintering and grain growth of NPLs, ${ }^{[51,52]}$ and 2) ligand-induced tetragonal lattice distortion which leads to a strain-induced change of bandgap and the expansion of the quantum well in the direction of short axis. ${ }^{[23,25,28]}$ As discussed above, there was no significant sintering or grain growth, but the expansion of quantum well thickness due to tetragonal lattice distortion was observed after $\mathrm{NH}_{4} \mathrm{Cl}$-treatment, implying that tetragonal lattice distortion was primary origin for the bathochromic shift (Figure 2a,c). The amount of bandgap redshift was roughly estimated based on lattice parameter change to be $152 \mathrm{meV}$, which was quite consistent with the energy shift of $142 \mathrm{meV}$ in absorption measurement (Figure S4 in the Supporting Information and discussion in the supporting information). Increased electronic coupling due to side-to-side attachment between NPLs could also contribute to this absorption peak shift and broadening. For the origin of emission quenching after $\mathrm{NH}_{4} \mathrm{Cl}$-treatment, one possible mechanism is charge transfer between NPLs due to enhanced electronic coupling enabled by significantly decreased side-to-side interplatelet distance or even side-to-side attachment (Figure 2a). ${ }^{[53]}$ Another possibility is that replacement of surface ligands creates in-gap states. ${ }^{[11]}$

To investigate the effects of replacement of surface ligands with $\mathrm{Cl}^{-}$on the electronic band structure of pristine, Cu-doped, and Ag-doped CdSe NPLs, density functional theory (DFT) analysis was conducted by modeling CdSe zinc blende NPLs with a periodic slab geometry consisting of $4 \mathrm{ML}$ perpendicular to the $\{100\}$ (Figure 3). For simplicity of the surface modeling, as-synthesized CdSe NPL was first studied with acetate ligand instead of oleic acid. ${ }^{[54]}$ It is noteworthy to point out that system is more stable when the acetates are attached on $\mathrm{Cd}$ atom in a zigzag manner than aligned one. The bandgap of acetate-capped pristine CdSe NPLs was calculated to be $2.08 \mathrm{eV}$, which is slightly smaller than the experimental value 
a)

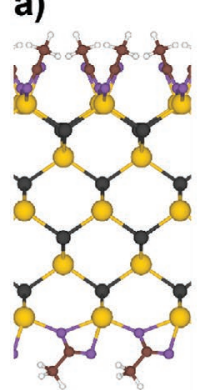

d)

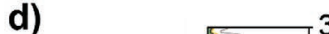

b)
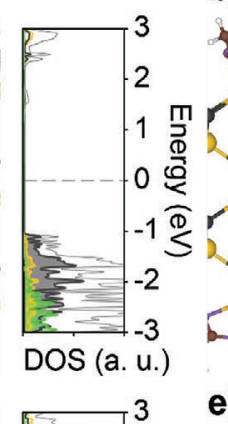

e)

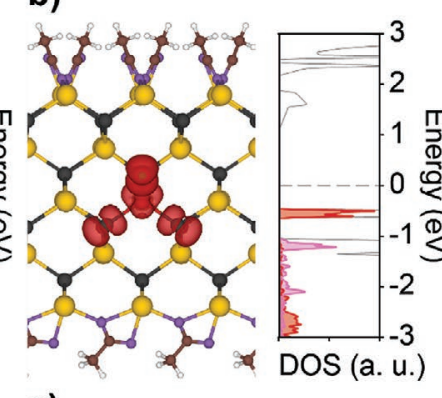

c)

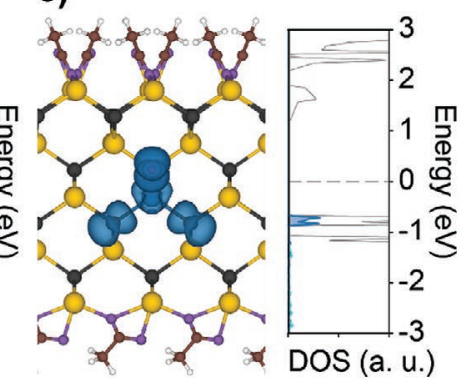

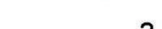

f)
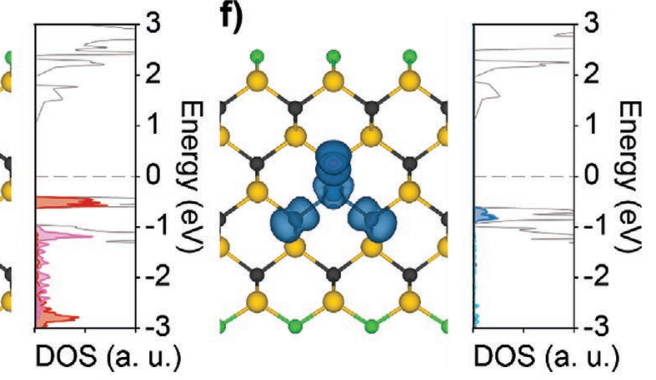

$\mathrm{Se} \bigcirc \mathrm{C} \bigcirc \mathrm{H} \bigcirc \mathrm{O} \bigcirc \mathrm{Cl}$

g) Oleate - CdSe NPL (Expt.)

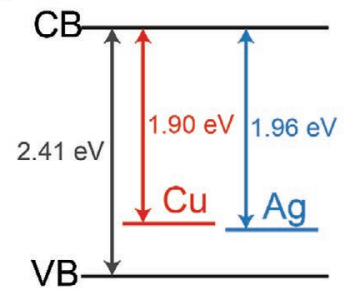

h) Acetate - CdSe NPL (DFT)
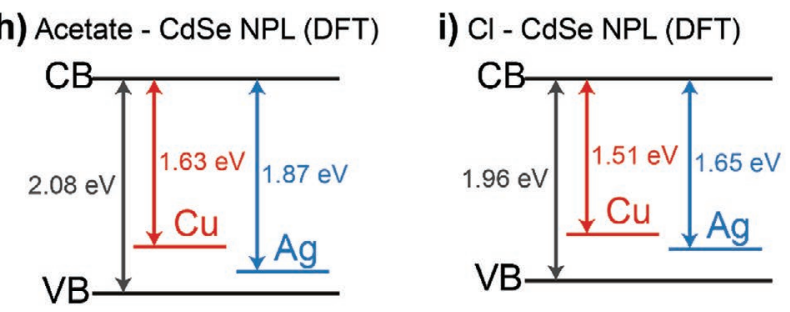

Figure 3. a) Schematic side view of acetate-capped pristine CdSe NPL and its calculated density of states. The charge densities of in-gap states and density of states of acetate-capped b) Cu-doped and c) Ag-doped CdSe NPL. d) Schematic side view of Cl-capped pristine CdSe NPL and its calculated density of states. The charge densities of in-gap states and density of states of Cl-capped e) Cu-doped and f) Ag-doped CdSe NPL. In (a) and (d), the density of states projected onto the $\mathrm{Cd}$, Se, and ligand are expressed with orange, grey, and green, respectively. The density of states projected onto the $t_{2 g}$ and $e_{g}$ orbitals of the $\mathrm{Cu}(\mathrm{Ag})$ are expressed with red (blue) and pink (cyan), respectively. The energy zero represents the midpoint between conduction band maximum (CBM) and valence band maximum (VBM). The charge density of in-gap states is drawn with an iso-surface of 0.005 electrons $\AA^{-3}$. Schematic representation of $\mathrm{Cu}$ - and Ag-induced energy states in the band structure of g) as-synthesized CdSe NPL based on g) optical analysis, h) acetate-capped and i) Cl-capped CdSe NPL based on DFT analysis.

from optical analysis (Figure 3a). For impurity doping, one $\mathrm{Cd}^{2+}$ per $2 \sqrt{2} \times 2 \sqrt{2}$ supercell was replaced with $\mathrm{Cu}^{+}$or $\mathrm{Ag}^{+}$to minimize the interaction between impurities. Energy of systems when $\mathrm{Cu}$ and $\mathrm{Ag}$ are positioned in middle, second, and surface layer of acetate-capped CdSe NPLs were calculated, respectively, showing that the most stable impurity position is the middle layer of NPLs (Table S1, Supporting Information). It was confirmed that the $t_{2 g}$ orbital of $\mathrm{Cu}$ and $\mathrm{Ag}$ and the $p$ orbital of the adjacent Se mainly forms a localized in-gap states as indicated by charge density and the density of state in Figure 3b,c. While $t_{2 g}$ state of the $\mathrm{Cu}$ was dominant in forming in-gap states in $\mathrm{Cu}$ doped CdSe NPLs, $p$ orbital of adjacent Se as well as $t_{2 g}$ state of the Ag contributed considerably in forming in-gap states in Agdoped CdSe NPLs. It is notable that $e_{\mathrm{g}}$ state of $\mathrm{Cu}$ was formed on the valence band edge, which is consistent with previous studies on CdSe nanocrystals. ${ }^{[37]}$ The energy difference from the conduction band edge to the defect state was found to be greater for Ag doping than $\mathrm{Cu}$ doping, which is consistent with the experimental results of optical analysis.
For the effect of $\mathrm{NH}_{4} \mathrm{Cl}$-ligand on their electronic band structure, CdSe NPLs with Cl ligand were studied using the same calculation method. The system was most stable when $\mathrm{Cl}$ was adsorbed to a bridge site between $\mathrm{Cd}$ atoms on the CdSe (001) plane (Figure 3d and Figure S5 (Supporting Information) and discussion in the supporting information). The bandgap of Cl-capped pristine CdSe NPLs was calculated to be $1.96 \mathrm{eV}$, which is slightly smaller than acetate-capped pristine CdSe NPLs due to reduced quantum confinement effect, further supporting that the absorption spectra change in CdSe $\mathrm{NPL}$ thin films after $\mathrm{NH}_{4} \mathrm{Cl}$-treatment mainly came from the tetragonal distortion of CdSe lattice. It should be noted that $\mathrm{Cl}$ states are positioned below the valence band maximum, while imperfect surface passivation with $\mathrm{Cl}$ or metastable adsorption of $\mathrm{Cl}$ on top of $\mathrm{Cd}$ could induce in-gap states (Figure S5 (Supporting Information) and discussion in the supporting information). ${ }^{[11]}$ Energy of systems depending on the position of impurities in Cl-capped CdSe NPLs were also investigated, showing that the most stable position of $\mathrm{Cu}$ and $\mathrm{Ag}$ is 
a)

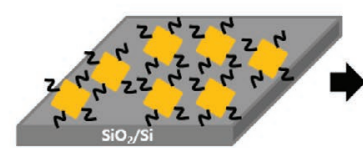

NPL coating

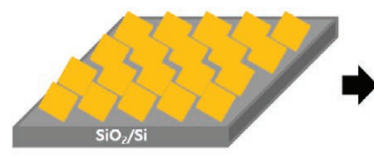

Ligand exchange \& annealing at $250^{\circ} \mathrm{C}$

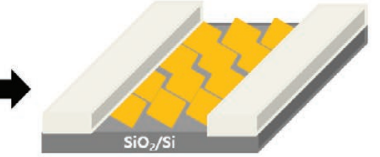

Thermal deposition of electrodes \& annealing at $250^{\circ} \mathrm{C}$
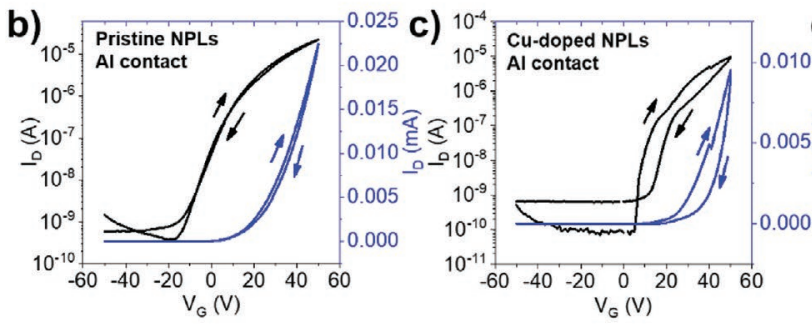

d)
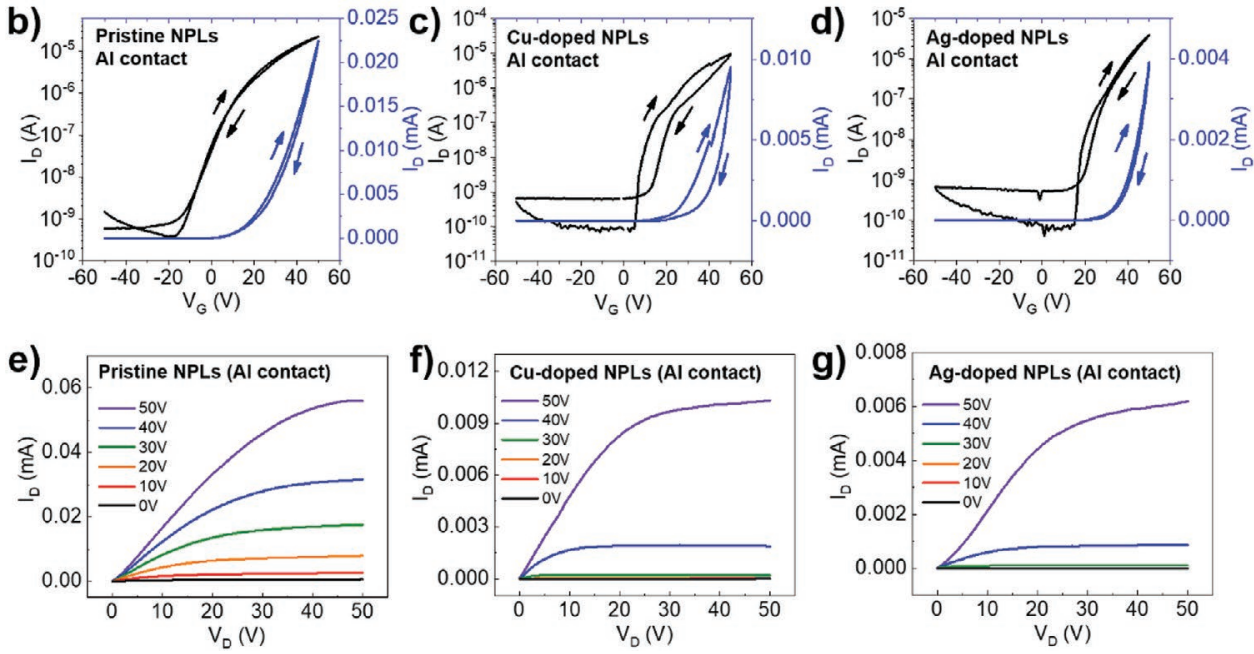

Figure 4. a) Schematic of process for fabrication of $\mathrm{NH}_{4} \mathrm{Cl}$-treated CdSe NPL FETs. Transfer characteristics $\left(I_{\mathrm{D}}-V_{\mathrm{C}}\right.$ at $\left.V_{\mathrm{D}}=50 \mathrm{~V}\right)$ for $\mathrm{NH}_{4} \mathrm{Cl}$-treated b) pristine, c) Cu-doped, and d) Ag-doped CdSe NPL FETs with Al electrodes. Output characteristics for $\mathrm{NH}_{4} \mathrm{Cl}$-treated e) pristine, f) $\mathrm{Cu}$-doped, and g) Ag-doped CdSe NPL FETs with Al electrodes.

the second layer and the middle layer, respectively (Table S1, Supporting Information). Although the bandgap of Cl-capped $\mathrm{Cu}$ - and Ag-doped CdSe NPLs was reduced compared to acetate-capped ones, the dopant induced in-gap state was still observed and the energy difference between conduction bandedge and dopant-induced state was still greater for Ag doping than $\mathrm{Cu}$ doping (Figure 3e,f). Thus, perfect Cl-passivation on a bridge site between $\mathrm{Cd}$ atoms does not significantly affect the position of $\mathrm{Cu}$ - and Ag-induced in-gap states and does not induce in-gap states (Figure $3 \mathrm{~g}-\mathrm{i}$ ). This implies that the significant quenching of band-edge and dopant-induced emissions is attributed to imperfect surface passivation with $\mathrm{Cl}$ or metastable adsorption of $\mathrm{Cl}$ on top of $\mathrm{Cd}$ as well as enhanced coupling between NPLs due to decreased side-to-side distance or even side-to-side attachment. Also, this enhanced interplatelet coupling might slightly contribute to the redshift and broadening of absorption.

To investigate the effects of $\mathrm{Cu}$ and Ag doping on electrical properties of CdSe NPLs, we fabricated field-effect transistors (FETs) based on a configuration of bottom gate and top contact with the following process (Figure 4a; Figure S6, Supporting Information): A heavily p-doped Si wafers with $285 \mathrm{~nm}$ thermally grown $\mathrm{SiO}_{2}$ were pre-cleaned and treated with MPTS to enhance NPL adhesion and minimize device hysteresis. Procedures of NPL spin-coating, ligand exchange, and washing with acetone were repeated twice respectively to form channel layers and the devices were annealed at $250{ }^{\circ} \mathrm{C}$ for $20 \mathrm{~min}$. $\mathrm{Al}$ electrodes were thermally deposited onto NPL channel layers to complete FET devices and the devices were briefly postannealed at $250{ }^{\circ} \mathrm{C}$. Without ligand exchange, all of the devices showed completely electrical insulating behavior and they did not even show any current modulation upon applied gate voltage because long original ligands lead to long interplatelet distance and extremely low charge transport efficiency or mobility (Figure S7, Supporting Information).

Representative transfer and output characteristics of $\mathrm{NH}_{4}$ Cl-treated pristine, Cu-doped, and Ag-doped CdSe NPL FETs are shown in Figure 4b-g. Saturation mobility $(\mu)$ of $(1.5 \pm 0.1) \times 10^{-1} \mathrm{~cm}^{2} \mathrm{~V}^{-1} \mathrm{~s}^{-1}$ and $I_{\mathrm{ON}} / I_{\mathrm{OFF}}$ of $(5.1 \pm 1.0) \times 10^{4}$ was achieved from $\mathrm{NH}_{4} \mathrm{Cl}$-treated pristine CdSe NPL thin films. Significantly improved mobility after $\mathrm{NH}_{4} \mathrm{Cl}$-treatment is originated from the reduced side-to-side distance. It should be noted that CdSe NPL thin films immersed in acetone for 2 min without $\mathrm{NH}_{4} \mathrm{Cl}$ still showed completely electrically insulating property as well as same absorption and emission features as as-synthesized CdSe NPL films, indicating that acetone did not alter optical and electrical properties (Figure S8, Supporting Information). In the case of $\mathrm{NH}_{4} \mathrm{Cl}$-treated $\mathrm{Cu}$-doped and Ag-doped CdSe NPL FETs, lower saturation mobility of $(9.1 \pm 1.7) \times 10^{-2} \mathrm{~cm}^{2} \mathrm{~V}^{-1} \mathrm{~s}^{-1}$ and $(8.7 \pm 0.4) \times 10^{-2} \mathrm{~cm}^{2} \mathrm{~V}^{-1} \mathrm{~s}^{-1}$ with $I_{\mathrm{ON}} / I_{\mathrm{OFF}}$ of $(1.3 \pm 0.2) \times 10^{4}$ and $(1.1 \pm 0.4) \times 10^{4}$ were observed, respectively. Both also showed a shift in the threshold voltage toward positive voltage, indicating lower electron concentration compared to pristine CdSe NPLs. Indeed, pristine CdSe NPL thin films showed higher electron concentration, mobility, and on/off ratio than $\mathrm{Cu}$-doped and Ag-doped NPL thin films. This implies that $\mathrm{Cu}$ and $\mathrm{Ag}$ dopants mitigate the n-type transport properties of CdSe NPLs. This is attributed to the formation of acceptor-like states by $\mathrm{Cu}$ and $\mathrm{Ag}$ dopants as confirmed by DFT and optical analysis, which compensates the intrinsic n-doping effects. It is known that Ag atoms could migrate under high potential, 

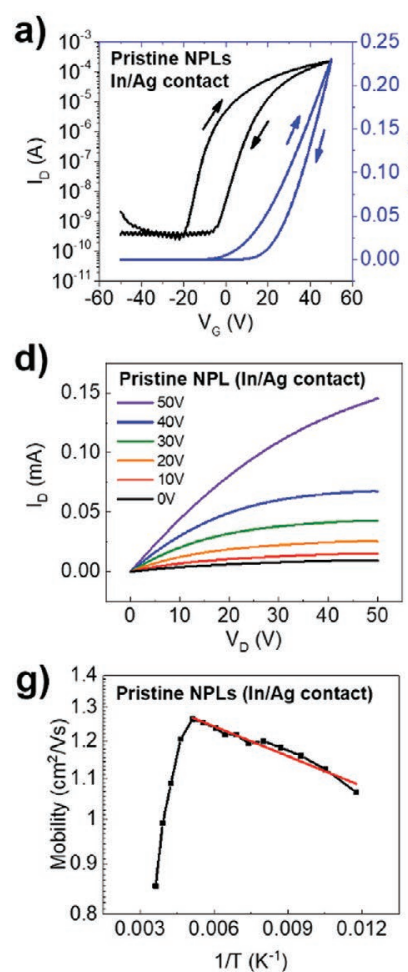

b)

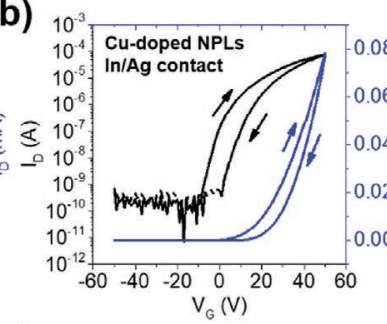

e)

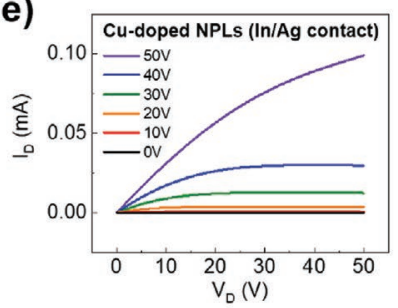

h)

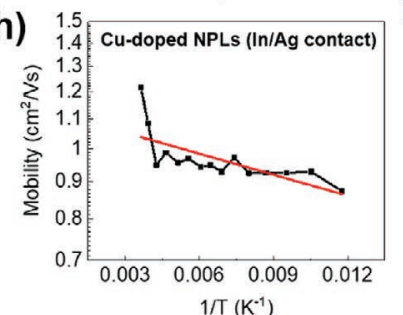

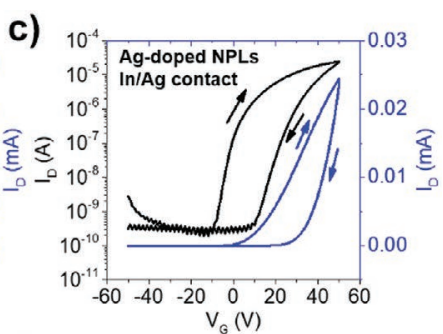

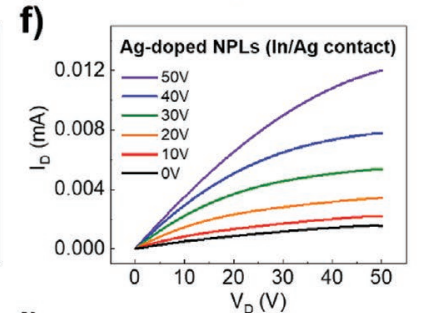

i)

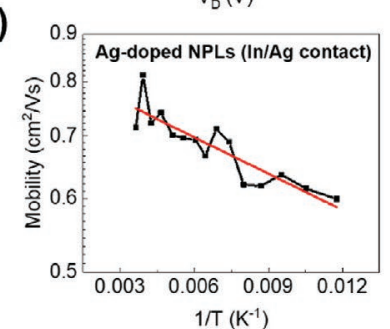

Figure 5. Transfer characteristics $\left(I_{D}-V_{\mathrm{C}}\right.$ at $\left.V_{\mathrm{D}}=50 \mathrm{~V}\right)$ for $\mathrm{NH}_{4} \mathrm{Cl}$-treated a) pristine, b) Cu-doped, and c) Ag-doped CdSe NPL FETs with In/Ag electrodes. Output characteristics for $\mathrm{NH}_{4} \mathrm{Cl}$-treated d) pristine, e) Cu-doped, and f) Ag-doped CdSe NPL FETs with In/Ag electrodes. Temperaturedependent mobilities of $\mathrm{NH}_{4} \mathrm{Cl}$-treated g) pristine, h) Cu-doped, and i) Ag-doped CdSe NPL TFTs with In/Ag electrodes from $85 \mathrm{~K}$ to $275 \mathrm{~K}$.

which could potentially affect transfer curves. To evaluate a potential migration of Ag dopants, we monitored a current in Ag-doped CdSe NPL thin films with Al electrodes as a function of time under $532 \mathrm{~nm}$ light $\left(3 \mathrm{~mW} \mathrm{~cm}^{-2}\right)$ by applying a pulsed voltage of 50 and $0 \mathrm{~V}$ (Figure S9, Supporting Information). A Photocurrent increased instantly to $1.2 \times 10^{-6} \mathrm{~A}$ upon applied $50 \mathrm{~V}$ and then slowly decayed to a saturation value of $3.0 \times 10^{-7} \mathrm{~A}$. When the applied voltage was removed, a negative current of $5.0 \times 10^{-9} \mathrm{~A}$ was immediately observed which decayed to zero. The current decay to a saturation value upon $50 \mathrm{~V}$ can be attributed to charge trapping or ion migration, but the negative current in the absence of external voltage can be only attributed to ion migration. ${ }^{[5,56]}$ However, the amount of current decay upon $50 \mathrm{~V}$ is higher by over two order of magnitude than the amount of negative current when the voltage was removed, suggesting ion migration effect is negligible compared to charge trapping.

Practical electronic applications generally require semiconducting materials with high mobility. ${ }^{[57]}$ Indium is known to effectively passivate defects of CdSe nanocrystals and act as n-type dopants in CdSe films. ${ }^{[35,58]}$ Thus, we replaced Al top contact with In/Ag top contact and briefly annealed the devices at $250{ }^{\circ} \mathrm{C}$ to induce diffusion of In into CdSe films and dope CdSe NPLs with In, increasing carrier concentration and improving charge transport efficiency. $\mathrm{NH}_{4} \mathrm{Cl}$-treated pristine, $\mathrm{Cu}$-doped, and Ag-doped CdSe NPL FETs fabricated with In/Ag electrodes showed saturation mobility of $1.44 \pm 0.43 \mathrm{~cm}^{2} \mathrm{~V}^{-1} \mathrm{~s}^{-1}$, $1.07 \pm 0.54 \mathrm{~cm}^{2} \mathrm{~V}^{-1} \mathrm{~s}^{-1}$, and $0.73 \pm 0.46 \mathrm{~cm}^{2} \mathrm{~V}^{-1} \mathrm{~s}^{-1}$ with $I_{\mathrm{ON}} /$ $I_{\mathrm{OFF}}$ of $(2.4 \pm 1.7) \times 10^{5},(1.5 \pm 0.6) \times 10^{5}$, and $(1.1 \pm 0.8) \times 10^{5}$, respectively (Figure 5a-f). A threshold shift toward negative voltage, higher mobility and $I_{\mathrm{ON}} / I_{\mathrm{OFF}}$ were observed in all series of CdSe NPL thin films when the devices were fabricated with $\mathrm{In} / \mathrm{Ag}$ electrodes compared to devices with $\mathrm{Al}$ electrodes. This implies that indium effectively n-dopes 2D CdSe NPLs. Pristine CdSe NPL FETs with In/Ag electrodes showed slightly lower mobilities by one order of magnitude and similar $I_{\mathrm{ON}} / I_{\mathrm{OFF}}$ compared to pristine CdSe quantum dot based FETs prepared with similar ligand exchange and In doping process from previous reports. ${ }^{[35,58-60]}$ It is notable that Cu-doped and Ag-doped NPL thin films showed a threshold voltage closer to zero and lower mobility compared to pristine NPLs, implying that $\mathrm{Cu}$ and Ag still mitigate n-type behaviors even after In doping. FETs with In/Ag electrodes showed larger hysteresis compared to devices with Al electrodes. Typical hysteresis of quantum dot based FETs comes from trapped carriers screening gate bias. ${ }^{[61]}$ In electrodes lower the injection barrier as well as increases charge transport via doping, which may lead to higher screening of gate bias and thus larger hysteresis..$^{[58,62]}$

In order to further evaluate the effects of $\mathrm{Cu}$ and $\mathrm{Ag}$ doping on charge transport in CdSe NPL thin films, temperature-dependent electrical characterization was conducted on pristine, Cu-doped, and Ag-doped CdSe NPL FETs with In/Ag electrodes in the range of $85 \mathrm{~K}-275 \mathrm{~K}$ (Figure $5 \mathrm{~g}-\mathrm{i}$; Figure S10, Supporting Information). In the case of pristine CdSe NPL FETs, mobility increased as temperature increases from $85 \mathrm{~K}$ to $195 \mathrm{~K}$ by following thermally activated transport mechanism 
$\mu=\mu_{0} \exp \left(-\frac{E_{\mathrm{A}}}{k T}\right)$

where $\mu_{0}$ is the pre-exponential factor, $E_{\mathrm{A}}$ is the activation energy for charge transport, $k$ is the Boltzmann constant, and $T$ is the temperature in Kelvin. For thermally activated transport, sufficient energy is needed to activate carriers from trap states as well as for carriers to hop between NPLs. ${ }^{[63]}$ From the slope of Arrhenius plots of mobility, $E_{\mathrm{A}}$ for pristine CdSe NPL FETs was extracted to be $2.01 \mathrm{meV}$ (Figure $5 \mathrm{~g}$ ). On the other hand, when temperature increases over $195 \mathrm{~K}$, opposite tendency against thermally activated transport, so-called band-like transport behavior, was observed in pristine CdSe NPL FETs. This might be attributed to extended electronic states due to decreased side-to-side distance or side-to-side attachment after $\mathrm{NH}_{4} \mathrm{Cl}$-treatment as well as effectively passivated trap states by In doping. ${ }^{[35,58]}$ In contrast, only thermally activated transport behavior was observed for $\mathrm{Cu}$ - and Ag-doped CdSe NPL FETs with $\mathrm{E}_{\mathrm{A}}$ of 1.9 and $2.5 \mathrm{meV}$, respectively, over the entire range of temperature (Figure $5 \mathrm{~h}, \mathrm{i})$. Considering that all series of $\mathrm{CdSe}$ NPLs have similar geometry and they were treated with the same ligand, and integrated into FETs with the same process, we believe the difference of charge transport behavior mainly stems from the effects of $\mathrm{Ag}$ and $\mathrm{Cu}$ doping, which induces in-gap states in CdSe NPL as confirmed by PL and DFT analysis. These dopant-induced in-gap states capture electrons and hinder efficient charge transport, leading to the observation of thermally activated transport behavior over the entire range of temperature. This demonstrates that $\mathrm{Cu}$ and $\mathrm{Ag}$ doping significantly affect not only optical properties, but also electrical properties and charge transport behaviors, providing important information for the design of impurity-doped nanomaterials and their optoelectronic device applications. However, further analysis of CdSe NPLs with higher doping concentration might be necessary to fully reveal the potential of the dopant.

\section{Conclusion}

In conclusion, we showed the effects of halide-ligand exchange and $\mathrm{Cu}$ and $\mathrm{Ag}$ doping on structural, chemical, optical, electronic, and electrical properties of four monolayer CdSe NPL using various characterization techniques. Side-to-side attachment between NPLs, tetragonal lattice distortion, bathochromic shift in absorption spectra, and complete quenching of bandedge and dopant-induced emissions were observed after $\mathrm{NH}_{4} \mathrm{Cl}$-treatment. Origin of each observation was systematically identified and understood by correlating with each other. First-principle calculations were conducted to fully understand the effects of Cl-passivation on the electronic band structure of pristine and impurity-doped CdSe NPLs. Field-effect transistors were fabricated to investigate electrical properties and charge transport behavior of pristine and impurity-doped CdSe NPLs, demonstrating $\mathrm{Cu}$ and $\mathrm{Ag}$ dopants mitigate $\mathrm{n}$-type character of CdSe NPLs. This work provides not only scientifically important and technologically useful information about surface chemistry and impurity doping of such semiconductor quantum wells, but also a new insight for the design of these nanomaterials for electronic and optoelectronic devices.

\section{Experimental Section}

Materials: Cadmium nitrate tetrahydrate, sodium myristate, technical grade 1-octadecene (ODE), selenium, cadmium acetate dihydrate, copper (II) acetate, silver acetate, trioctylphosphine (TOP), oleylamine, ammonium sulfde, $\mathrm{N}$-methylformamide, and technical grade oleic acid (OA), (3-mercaptopropyl)trimethoxysilane (MPTS) (95\%), isopropanol (99.5\%), acetone $(\geq 99.9 \%)$, ammonium chloride $\left(\mathrm{NH}_{4} \mathrm{Cl}, 99.99 \%\right)$ were purchased from Sigma-Aldrich. Methanol, ethanol, acetone, and hexane were purchased from Merck Millipore. All reagents were used without further purification.

Synthesis of Pristine, Cu-Doped, and Ag-Doped CdSe NPLs: All NPLs were synthesized by following previous reported recipe. ${ }^{[41,42]}$ For $4 \mathrm{ML}$ of Cu-doped NPL synthesis, $0.34 \mathrm{~g}$ of cadmium myristate, $0.024 \mathrm{~g}$ of Se, and $30 \mathrm{~mL}$ of ODE were loaded into a $100 \mathrm{~mL}$ three-neck flask. The solution was degassed and stirred at $95{ }^{\circ} \mathrm{C}$ under vacuum for $1 \mathrm{~h}$, and the temperature controller was set to $240{ }^{\circ} \mathrm{C}$ under argon gas. As the temperature reached $190{ }^{\circ} \mathrm{C}, 0.04 \mathrm{~mL}$ of $\mathrm{Cu}$ precursor solution (prepared with $15 \mathrm{mg}$ of copper (II) acetate, $2 \mathrm{~mL}$ of TOP, and $5 \mathrm{~mL}$ of ODE) was added dropwise and $120 \mathrm{mg}$ of cadmium acetate dihydrate was introduced swiftly. After $10 \mathrm{~min}, 1 \mathrm{~mL}$ of OA was injected and the temperature of the solution was decreased to room temperature. The solution was centrifuged for $5 \mathrm{~min}$ at $6000 \mathrm{rpm}$, and ethanol was added into the supernatant solution until it became turbid, centrifuged at $10000 \mathrm{rpm}$ for $5 \mathrm{~min}$. The precipitates were dissolved and stored in toluene. Undoped $4 \mathrm{ML}$ CdSe NPLs were synthesized by injecting bare TOP-ODE solutions with the same conditions used for preparing $\mathrm{Cu}$ precursor.

For Ag-doped CdSe NPL synthesis, $340 \mathrm{mg}$ of cadmium myristate, $24 \mathrm{mg}$ of Se, and $30 \mathrm{~mL}$ of ODE were added in a $100 \mathrm{~mL}$ flask. The solution as degassed and stirred for $1 \mathrm{~h}$ at $95{ }^{\circ} \mathrm{C}$, and the temperature of the solution was raised to $240{ }^{\circ} \mathrm{C}$. The $\mathrm{Ag}$ precursor (prepared with $30 \mathrm{mg}$ of silver acetate, $3.6 \mathrm{~mL}$ of TOP, and $0.4 \mathrm{~mL} \mathrm{ODE}$ ) was injected at $190^{\circ} \mathrm{C}$ and the temperature of the solution was lowered to $90^{\circ} \mathrm{C}$ by cooling with a water bath. The solution was degassed under vacuum for few minutes and heated to $240{ }^{\circ} \mathrm{C}$ under argon atmosphere. At $130^{\circ} \mathrm{C}$ $120 \mathrm{mg}$ of cadmium acetate was introduced, and the reaction solution was maintained at $240{ }^{\circ} \mathrm{C}$ for $10 \mathrm{~min}$. Then $1 \mathrm{~mL}$ of OA were injected, and the solution was cooled to room temperature and centrifuged at $6000 \mathrm{rpm}$ for $5 \mathrm{~min}$ for the purification.

Substrate Preparation: Glass substrates were used for the UV-vis, PL, and FTIR spectroscopy measurements. Silicon wafer was used for XRD analysis. Heavily p-doped silicon wafers with a thermally grown $\mathrm{SiO}_{2}$ layer ( $285 \mathrm{~nm}$ thick) were used for the FET measurements. All substrates were washed with acetone, followed by isopropanol and deionized water, with sonication. The substrates were treated with UV-ozone and finally immersed in 5 vol\% MPTS in toluene overnight.

Device Fabrication: To fabricate FETs, the CdSe NPLs were spin-coated onto a pre-cleaned and MPTS-treated $\mathrm{Si} / \mathrm{SiO}_{2}$ wafer. The samples were immersed in $\mathrm{NH}_{4} \mathrm{Cl}$ solution in acetone for ligand exchange and rinsed with acetone. The NPL spin-coating, ligand exchange, and washing procedures were repeated once again, and the samples were annealed at $250^{\circ} \mathrm{C}$ for $20 \mathrm{~min}$. Thereafter, an $\mathrm{Al}(60 \mathrm{~nm})$ or $\mathrm{In} / \mathrm{Ag}(50 \mathrm{~nm} / 50 \mathrm{~nm})$ top contact was deposited by thermal evaporation. The devices were then post-annealed at $250^{\circ} \mathrm{C}$ for $5 \mathrm{~min}$ under nitrogen. The ratio of the channel width (W) to length (L) (i.e., $W / L$ ) was 15 for all devices. Each device was mechanically isolated from the edge of the substrate and from the other devices.

Measurement and Characterization: FET characterization was conducted using a semiconductor parameter analyzer (Model 4200ASCS, KEITHLEY) in a nitrogen glove box. Temperature-dependent electrical characterization was performed using the same parameter analyzer and a low-temperature vacuum probe station (Model M5VC, MSTECH). Transmission electron microscopy (Model Tecnai G2 F30, FEI, Korea Basic Science Institute) and XRD (Model SmartLab, Rigaku) was used to characterize the structural properties of the CdSe NPL thin films. Absorption spectra of Cu-doped CdSe NPLs in hexane was measured by a UV-vis spectrophotometer (Shimadzu, UV-1800). PL 
spectra of Cu-doped CdSe NPLs in hexane were recorded using a fibercoupled ANDOR spectrometer (monochromator: ANDOR Shamrock 303i, CCD: ANDOR iDus 401) under a diode laser excitation (Cobolt 06-MLD, excitation wavelength: $405 \mathrm{~nm}$ ). The optical and chemical properties of the CdSe NPL thin films were characterized by UV-vis spectroscopy (Model Cary 5000, Agilent Technologies), PL spectroscopy (FP-8500, JASCO Corporation) and FTIR spectroscopy (Model LabRam ARAMIS IR2, Horiba Jobin Yvon). For ICP-MS measurements, $100 \mu \mathrm{L}$ solution of NPLs was precipitated with excess ethanol and dissolved with $2 \%$ of $65 \% \mathrm{HNO}_{3}$, which was added to digest the NPL for ICP measurements. ICP elemental calibration curves for $\mathrm{Cd}, \mathrm{Cu}$, and Se were created using commercial standards with known concentrations.

Computational Methods: Our first-principles DFT calculations were performed using the Vienna ab initio simulation package (VASP) employing projector-augmented wave potentials that describe the interaction between ion cores and valence electrons. ${ }^{[64-66]}$ Exchange-correlation energy was treated using the GGA functional of Perdew-Burke-Ernzerhof (PBE) as well as the hybrid functional of HeydScuseria-Ernzerhof (HSE06). ${ }^{[67-69]}$ The HSE06 functional is given by

$$
\begin{aligned}
E_{X C}^{H S E 06}= & 1 / 4 E_{X}^{H F, S R}\left(\omega<0.2 \AA^{-1}\right)+3 / 4 E_{X}^{P B E, S R}\left(\omega<0.2 \AA^{-1}\right. \\
& +E_{X}^{P B E, L R}\left(\omega>0.2 \AA^{-1}\right)+E_{C}^{P B E}
\end{aligned}
$$

where the screening parameter $\omega\left(0.2 \AA^{-1}\right)$ defines the separation of short range (SR) and long range (LR) for the exchange energy. The plane wave basis was used with a kinetic energy cutoff of 600 and $400 \mathrm{eV}$ for PBE and HSEO6 functionals, respectively. The $k$-space integrations were done with the $3 \times 3 k$ points for $2 \sqrt{2} \times 2 \sqrt{2}$ supercell. The CdSe NPLs were modeled by a periodic slab geometry consisting of the $\approx 15 \AA$ of vacuum in between the slabs. All atoms were allowed to relax along the calculated forces until all the residual force components were smaller than $5 \mathrm{meV} / \AA$ for PBE functional. In the HSE06 calculation, optimized structure obtained by PBE calculation was used. The bandgap, partial density of states, and partial charge densities of in-gap states are the results of HSEO6 calculations.

\section{Supporting Information}

Supporting Information is available from the Wiley Online Library or from the author.

\section{Acknowledgements}

W.S.L. and Y.-G.K. contributed equally to this work. The authors would like to acknowledge the financial support from Singapore National Research Foundation under the Program of NRF-NRFI2016-08, the Competitive Research Program NRF-CRP14-2014-03, and Singapore Ministry of Education Tier 1 grant (MOE-RG62/20). The authors acknowledge the financial support from National Science Foundation the Basic Science Research Program through the National Research Foundation of Korea (NRF) funded by the Ministry of Science, ICT and Future Planning (2019R1C1C1003319), Creative Materials Discovery Program through the National Research Foundation of Korea (NRF) funded by Ministry of Science, ICT and Future Planning (NRF2018M3D1A1059001), and Korea University Future Research Grant. H.V.D. also acknowledges support from TUBA. Y.G.K. and M.J.H. were supported by the Basic Science Research Program through the National Research Foundation of Korea (NRF) funded by the Ministry of Science and ICT (2021R1A2C1009303).

\section{Conflict of Interest}

The authors declare no conflict of interest.

\section{Data Availability Statement}

The data that support the findings of this study are available from the corresponding author upon reasonable request.

\section{Keywords}

charge transport, doping, field-effect transistors, ligand exchange, nanoplatelets

Received: July 20, 2021

Revised: August 25, 2021

Published online: September 23, 2021

[1] M. Sharma, S. Delikanli, H. V. Demir, Proc. IEEE 2020, 108, 655

[2] S. K. Ha, C. M. Mauck, W. A. Tisdale, Chem. Mater. 2019, 31, 2486.

[3] S. Ithurria, M. D. Tessier, B. Mahler, R. P. S. M. Lobo, B. Dubertret, A. L. Efros, Nat. Mater. 2011, 10, 936.

[4] M. C. Weidman, M. Seitz, S. D. Stranks, W. A. Tisdale, ACS Nano 2016, 10, 7830.

[5] X. Liu, D. Yu, F. Cao, X. Li, J. Ji, J. Chen, X. Song, H. Zeng, Small 2017, 13, 1700364

[6] M. C. Weidman, A. J. Goodman, W. A. Tisdale, Chem. Mater. 2017, 29, 5019.

[7] S. Delikanli, G. Yu, A. Yeltik, S. Bose, T. Erdem, J. Yu, O. Erdem, M. Sharma, V. K. Sharma, U. Quliyeva, S. Shendre, C. Dang, D. H. Zhang, T. C. Sum, W. Fan, H. V. Demir, Adv. Funct. Mater. 2019, 29, 1901028.

[8] Z. Chen, B. Nadal, B. Mahler, H. Aubin, B. Dubertret, Adv. Funct. Mater. 2014, 24, 295.

[9] W. -K. Koh, N. K. Dandu, A. F. Fidler, V. I. Klimov, J. M. Pietryga, S. V. Kilina, J. Am. Chem. Soc. 2017, 139, 2152.

[10] Y. Gao, M. C. Weidman, W. A. Tisdale, Nano Lett. 2017, 17, 3837

[11] S. Singh, R. Tomar, S. ten Brinck, J. de Roo, P. Geiregat, J. C. Martins, I. Infante, Z. Hens, J. Am. Chem. Soc. 2018, 140, 13292.

[12] J. Liu, L. Guillemeney, B. Abécassis, L. Coolen, Nano Lett. 2020, 20, 3465.

[13] O. Erdem, S. Foroutan, N. Gheshlaghi, B. Guzelturk, Y. Altintas, H. V. Demir, Nano Lett. 2020, 20, 6459.

[14] E. Lhuillier, S. Pedetti, S. Ithurria, H. Heuclin, B. Nadal, A. Robin, G. Patriarche, N. Lequeux, B. Dubertret, ACS Nano 2014, 8, 3813.

[15] E. Lhuillier, J.-F. Dayen, D. O. Thomas, A. Robin, S. Ithurria, H. Aubin, B. Dubertret, Phys. Status SolidiC 2016, 13, 526.

[16] E. Lhuillier, S. Ithurria, A. Descamps-Mandine, T. Douillard, R. Castaing, X. Zhen Xu, P.-L. Taberna, P. Simon, H. Aubin, B. Dubertret, J. Phys. Chem. C 2015, 119, 21795.

[17] E. Lhuillier, J.-F. Dayen, D. O. Thomas, A. Robin, B. Doudin, B. Dubertret, Nano Lett. 2015, 15, 1736.

[18] D. K. Kim, A. T. Fafarman, B. T. Diroll, S. H. Chan, T. R. Gordon, C. B. Murray, C. R. Kagan, ACS Nano 2013, 7, 8760.

[19] Y. Liu, M. Gibbs, J. Puthussery, S. Gaik, R. Ihly, H. W. Hillhouse, M. Law, Nano Lett. 2010, 10, 1960.

[20] S. J. Oh, N. E. Berry, J. H. Choi, E. A. Gaulding, T. Paik, S. H. Hong, C. B. Murray, C. R. Kagan, ACS Nano 2013, 7, 2413.

[21] W. S. Lee, S. W. Lee, H. Joh, M. Seong, H. Kim, M. S. Kang, K. H. Cho, Y. M. Sung, S. J. Oh, Small 2017, 13, 1702534.

[22] S. Choi, J. Park, W. Hyun, J. Kim, J. Kim, Y. B. Lee, C. Song, H. J. Hwang, J. H. Kim, T. Hyeon, D. H. Kim, ACS Nano 2015, 9, 6626.

[23] A. Antanovich, A. W. Achtstein, A. Matsukovich, A. Prudnikau, P. Bhaskar, V. Gurin, M. Molinari, M. Artemyev, Nanoscale 2017, 9, 18042. 
[24] C. Ji, W. E. Buhro, 2020, 32, 5290.

[25] B. T. Diroll, Chem. Mater. 2020, 32, 5916.

[26] S. Jana, M. de Frutos, P. Davidson, B. Abécassis, Sci. Adv. 2017, 3, 1701483.

[27] M. Dufour, J. Qu, C. Greboval, C. M. Méthivier, E. Lhuillier, S. Ithurria, ACS Nano 2019, 13, 5326.

[28] B. T. Diroll, R. D. Schaller, Chem. Mater. 2019, 31, 3556.

[29] H. Sun, W. E. Buhro, Chem. Mater. 2020, 32, 5814.

[30] T. Jiang, J. Song, H. Wang, X. Ye, H. Wang, W. Zhang, M. Yang, R. Xia, L. Zhu, X. Xu, J. Mater. Chem. B 2015, 3, 2402.

[31] K. Xing, X. Yuan, Y. Wang, J. Li, Y. Wang, Y. Fan, L. Yuan, K. Li, Z. Wu, H. Li, J. Zhao, J. Phys. Chem. Lett. 2019, 10, 4177.

[32] H. Lu, G. M. Carroll, X. Chen, D. K. Amarasinghe, N. R. Neale, E. M. Miller, P. C. Sercel, F. A. Rabuffetti, A. L. Efros, M. C. Beard, J. Am. Chem. Soc. 2018, 140, 13753.

[33] S. C. Erwin, L. Zu, M. I. Haftel, A. L. Efros, T. A. Kennedy, D. J. Norris, Nature 2005, 436, 91.

[34] H. Luo, C. Tuinenga, E. B. Guidez, C. Lewis, J. Shipman, S. Roy, C. M. Aikens, V. Chikan, J. Phys. Chem. C 2015, 119, 10749.

[35] W. S. Lee, Y. G. Kang, H. K. Woo, J. Ahn, H. Kim, D. Kim, S. Jeon, M. J. Han, J. H. Choi, S. J. Oh, Chem. Mater. 2019, 31, 9389.

[36] A. Sahu, M. S. Kang, A. Kompch, C. Notthoff, A. W. Wills, D. Deng, M. Winterer, C. D. Frisbie, D. J. Norris, Nano Lett. 2012, 12, 2587.

[37] H. D. Nelson, S. O. M. Hinterding, R. Fainblat, S. E. Creutz, X. Li, D. R. Gamelin, J. Am. Chem. Soc. 2017, 139, 6411.

[38] L. Yang, K. E. Knowles, A. Gopalan, K. E. Hughes, M. C. James, D. R. Gamelin, Chem. Mater. 2016, 28, 7375.

[39] M. Sharma, M. Olutas, A. Yeltik, Y. Kelestemur, A. Sharma, S. Delikanli, B. Guzelturk, K. Gungor, J. R. McBride, H. V. Demir, Chem. Mater. 2018, 30, 3265.

[40] A. H. Khan, V. Pinchetti, I. Tanghe, Z. Dang, B. Martín-García, Z. Hens, D. van Thourhout, P. Geiregat, S. Brovelli, I. Moreels, Chem. Mater. 2019, 31, 1450.

[41] M. Sharma, K. Gungor, A. Yeltik, M. Olutas, B. Guzelturk, Y. Kelestemur, T. Erdem, S. Delikanli, J. R. McBride, H. V. Demir, Adv. Mater. 2017, 29, 1700821.

[42] A. Najafi, M. Sharma, S. Delikanli, A. Bhattacharya, J. R. Murphy, J. Pientka, A. Sharma, A. P. Quinn, O. Erdem, S. Kattel, Y. Kelestemur, M. V. Kovalenko, W. D. Rice, H. V. Demir, A. Petrou, J. Phys. Chem. Lett. 2021, 12, 2892.

[43] G. H. V. Bertrand, A. Polovitsyn, S. Christodoulou, A. H. Khan, I. Moreels, Chem. Commun. 2016, 52, 11975.

[44] J. Yu, M. Sharma, M. Li, S. Delikanli, A. Sharma, M. Taimoor, Y. Altintas, J. R. McBride, T. Kusserow, T. C. Sum, H. V. Demir, C. Dang, Laser Photonics Rev. 2021, 15, 2100034.

[45] P. Pallavicini, G. Dacarro, M. Galli, M. Patrini, J. Colloid Interface Sci. 2009, 332, 432.

[46] I. Fedin, D. v. Talapin, J. Am. Chem. Soc. 2016, 138, 9771.
[47] E. Marino, T. E. Kodger, R. W. Crisp, D. Timmerman, K. E. MacArthur, M. Heggen, P. Schall, Angew. Chem. 2017, 129, 13983.

[48] A. Riedinger, F. D. Ott, A. Mule, S. Mazzotti, P. N. Knüsel, S. J. P. Kress, F. Prins, S. C. Erwin, D. J. Norris, Nat. Mater. 2017, $16,743$.

[49] J. Rockenberger, L. Troger, A. L. Rogach, M. Tischer, M. Grundmann, A. Eychmuller, H. Weller, J. Chem. Phys. 1998, 108, 7807.

[50] A. Antanovich, A. Prudnikau, A. Matsukovich, A. Achtstein, M. Artemyev, J. Phys. Chem. C 2016, 120, 5764.

[51] M. J. Greaney, E. Couderc, J. Zhao, B. A. Nail, M. Mecklenburg, W. Thornbury, F. E. Osterloh, S. E. Bradforth, R. L. Brutchey, Chem. Mater. 2015, 27, 744.

[52] Z. M. Norman, N. C. Anderson, J. S. Owen, ACS Nano 2014, 8, 7513.

[53] M. Wu, P. Mukherjee, D. N. Lamont, D. H. Waldeck, J. Phys. Chem. C 2010, 114, 5751.

[54] R. S. Koster, C. Fang, A. van Blaaderen, M. Dijkstra, M. A. van Huis, Phys. Chem. Chem. Phys. 2016, 18, 22021.

[55] L. Flannery, J. Ogle, D. Powell, C. Tassone, L. Whittaker-Brooks, J. Mater. Chem. A 2020, 8, 25109.

[56] D. Li, H. Wu, H. C. Cheng, G. Wang, Y. Huang, X. Duan, ACS Nano 2016, 10, 6933

[57] S.-K. Su, C.-P. Chuu, M.-Y. Li, C.-C. Cheng, H.-S. P. Wong, L.-J. Li, Small Structures 2021, 2, 2000103.

[58] J. H. Choi, A. T. Fafarman, S. J. Oh, D. K. Ko, D. K. Kim, B. T. Diroll, S. Muramoto, J. G. Gillen, C. B. Murray, C. R. Kagan, Nano Lett. 2012, 12, 2631.

[59] V. Sayevich, C. Guhrenz, M. Sin, V. M. Dzhagan, A. Weiz, D. Kasemann, E. Brunner, M. Ruck, D. R. T. Zahn, K. Leo, N. Gaponik, A. Eychmüller, Adv. Funct. Mater. 2016, 26, 2163.

[60] S. M. Jung, H. L. Kang, J. K. Won, J. Kim, C. Hwang, K. Ahn, I. Chung, B. K. Ju, M. G. Kim, S. K. Park, ACS Appl. Mater. Interfaces 2018, 10, 3739.

[61] M. S. Kang, A. Sahu, D. J. Norris, C. D. Frisbie, Nano Lett. 2010, 10, 3727.

[62] A. T. Fafarman, W. K. Koh, B. T. Diroll, D. K. Kim, D. K. Ko, S. J. Oh, X. Ye, V. Doan-Nguyen, M. R. Crump, D. C. Reifsnyder, C. B. Murray, C. R. Kagan, J. Am. Chem. Soc. 2011, 133, 15753.

[63] M. S. Kang, A. Sahu, D. J. Norris, C. D. Frisbie, Nano Lett. 2010, 10, 3727.

[64] G. Kresse, J. Hafner, Phys. Rev. B 1993, 48, 13115.

[65] G. Kresse, J. Furthmüller, Comput. Mater. Sci. 1996, 6, 15.

[66] G. Kresse, D. Joubert, Phys. Rev. B: Condens. Matter Mater. Phys. 1999, 59, 1758.

[67] J. P. Perdew, K. Burke, M. Ernzerhof, Phys. Rev. Lett. 1996, 77, 3865.

[68] J. Heyd, G. E. Scuseria, M. Ernzerhof, J. Chem. Phys. 2003, 118, 8207.

[69] A. V. Krukau, O. A. Vydrov, A. F. Izmaylov, G. E. Scuseria, J. Chem. Phys. 2006, 125, 224106. 